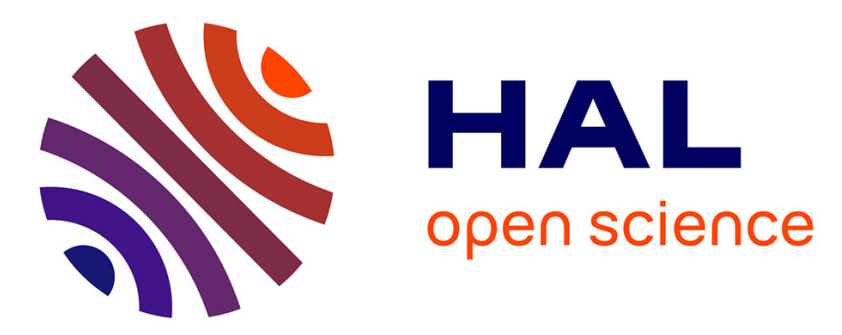

\title{
De l'union de l'âme et du corps à l'unité de la sensibilité. L'anthropologie méconnue de L'Esprit des lois
}

Céline Spector

\section{To cite this version:}

Céline Spector. De l'union de l'âme et du corps à l'unité de la sensibilité. L'anthropologie méconnue de L'Esprit des lois. Les études philosophiques, 2013, 106 (3), pp.383-396. 10.3917/leph.133.0383 . hal-02476036

\section{HAL Id: hal-02476036 \\ https://hal.sorbonne-universite.fr/hal-02476036}

Submitted on 17 Feb 2020

HAL is a multi-disciplinary open access archive for the deposit and dissemination of scientific research documents, whether they are published or not. The documents may come from teaching and research institutions in France or abroad, or from public or private research centers.
L'archive ouverte pluridisciplinaire HAL, est destinée au dépôt et à la diffusion de documents scientifiques de niveau recherche, publiés ou non, émanant des établissements d'enseignement et de recherche français ou étrangers, des laboratoires publics ou privés. 


\title{
De l'union de l'âme et du corps à l'unité de la sensibilité. L'anthropologie méconnue de L'Esprit des lois'.
}

\author{
On ne s'est jamais trompé plus \\ grossièrement que lorsqu'on a voulu \\ réduire en système les sentiments des \\ hommes (Mes Pensées, $\mathrm{n}^{\circ}$ 30).
}

Résumé: Montesquieu n'est pas considéré comme l'un des fondateurs de l'anthropologie entendue comme science de l'homme, mais comme le précurseur de la sociologie. Cette contribution entend discuter ce jugement : la science nouvelle de L'Esprit des lois repose sur une anthropologie de la force, et de l'unité de la sensibilité.

Summary : Montesquieu is not usually considered as one of the founding fathers of anthropology understood as the science of man, but as the forerunner of sociology. This paper discusses this view. The new science of The Spirit of the Laws relies on a theory of forces, and on the unity of sensibility.

En quel sens peut-on parler d'anthropologie au moment où écrit Montesquieu ? Définie comme la connaissance de l'homme physique et moral, l'anthropologie articule anatomie et psychologie ${ }^{2}$. D'un côté, l'anatomie conserve au XVIII siècle une importance majeure, ce qui transparaît dans l'Encyclopédie: "Anthropologie, dans l'aconomie animale: c'est un traité de l'homme ». De l'autre, la psychologie se métamorphose à la suite des bouleversements introduits par Descartes, Locke et Newton. A l'étude métaphysique du dualisme substantiel se substitue une recherche empiriste des interactions entre l'âme et le corps : le lieu de leur entrelacs - la sensibilité - est centralisé dans le cerveau mais diffus dans toutes les parties du corps par l'intermédiaire des nerfs. Enfin, l'anthropologie tente de cerner la spécificité de l'homme au sein du règne vivant et

1 «De l'union de l'âme et du corps à l'unité de la sensibilité : l'anthropologie méconnue de L'Esprit des lois », Les Etudes philosophiques, Juillet 2013, p. 383-396.

${ }^{2}$ Dans l'immense littérature consacrée à cette question, on mentionnera ici G. Gusdorf, Introduction aux sciences humaines. Essai critique sur leurs origines et leur développement, Paris, Ophrys, 1974 ; S. Moravia, La scienza dell'uomo nel Settecento, Bari, Laterza, 1970, et l'introduction qu'il consacre à la traduction italienne de G. Gusdorf, Le scienze umane nel secolo dei lumi, Florence, La Nuova Italia, 1980 (originellement : L'Avènement des sciences bumaines au siècle des Lumières, Paris, Payot, 1973). 
animal, et pose la question des variétés de l'espèce humaine. Les deux derniers sens convergeront à la fin du siècle, lors de la fondation des Sociétés d'anthropologie et sous l'impulsion des Idéologues ${ }^{3}$.

Or contrairement à Buffon ou à Rousseau, Montesquieu n'est jamais mentionné parmi les fondateurs de l'anthropologie. Il n'est pas invoqué par ses successeurs qui participent à l'émergence de l'anthropologie au XVIII ${ }^{\mathrm{e}}$ siècle, environ vingt ans après la parution de L'Esprit des lois. Lorsque J.-B. Robinet, auteur d'un Traité complet et systématique d'anthropologie, en mentionne des ébauches, il cite «le Traité de l'homme de M. de Buffon, l'Essai de psychologie de M. Bonnet, le Traité des sensations de M. l'abbé de Condillac, le Traité de l'bomme et de son éducation par Helvétius, et même l'anthropologie du Marquis de Gorini Corio ", mais pas L'Esprit des lois'. Considéré comme le fondateur de la sociologie, Montesquieu n'apparait pas davantage chez les historiens de l'anthropologie, quelles qu'en soient les tendances ${ }^{5}$. Pour M. Crépon, la " géographie de l'esprit » mise en place dans L'Esprit des lois relève non d'une enquête anthropologique mais d'une investigation historique ${ }^{6}$. Enfin, les exégèses les plus opposées de Montesquieu parviennent à une conclusion analogue. Selon P. Manent, qui s'inscrit dans le sillage de Leo Strauss, L'Esprit des lois sonne le glas de la perspective classique centrée sur la détermination d'une essence de l'homme ou d'une théorie de l'âme. La sociologie comme «science de l'homme » n'existerait qu'en refusant d'être science de la nature de l'homme ${ }^{7}$. Pour L. Althusser en revanche, Montesquieu serait en rupture avec la perspective moderne - celle d'une science de la nature humaine comme science de ses passions. L'Esprit des lois ne conçoit plus les lois du monde intellectuel ou moral comme des lois de la nature humaine, mais comme des lois de la nature des choses, induites des sociétés réelles ${ }^{8}$. En dernière instance, Montesquieu serait celui qui a rompu avec l'impératif, antique ou moderne, d'une anthropologie comme théorie de la nature de l'homme.

$\mathrm{Au}$ regard de ces lectures convergentes, il est donc légitime de s'interroger: l'anthropologie disparait-elle de L'Esprit des lois, et la " science nouvelle " qu'il fonde s'édifie-t-elle sur l'abandon de la science de l'homme? Cette contribution entend discuter ce jugement: il s'agira de mesurer les conséquences de la focalisation nouvelle sur les peuples comme sujets de l'histoire ; L'Esprit des lois fait advenir une histoire naturelle des hommes et d'émergence de leur sensibilité collective. La théorie de l'émergence de la sensibilité, des manières de penser et de sentir communes, fonde la possibilité d'une science politique centrée sur le concept original d'« esprit général » : «Plusieurs choses gouvernent les hommes, le climat, la religion, les lois, les maximes du gouvernement, les exemples des choses passées, les mœurs, les manières ; d'où il se forme un esprit général qui en résulte $»^{10}$. Mais une anthropologie de la force commande la dynamique de l'art politique.

3 G. Gusdorf, Dieu, la nature, l'homme au siècle des Lumières, Paris, Payot, 1972, p. 356 sq.

4 J.-B. Robinet, article "Anthropologie, ou traité de l'homme», in Dictionnaire universel des sciences morale, économique, politique et diplomatique, Londres, t. V, 1778, p. 334.

5 Montesquieu apparaît ici comme fondateur de la sociologie. Voir G. Gusdorf, Introduction aux sciences humaines, op. cit., en partic. p. 256 et, contre Gusdorf, M. Duchet, Anthropologie et Histoire au siècle des Lumières, Paris, Albin Michel, 1995, avec la postface de C. Blanckaert: "Les archives du genre humain. Approches réflexives en histoire des sciences anthropologiques »; R. Wolker, "Anthropology and Conjectural History in the Enlightenment », in Inventing Human Sciences. Eighteenth Century Domains, C. Fox, R. Porter et R. Wolker éds., Berkeley, University of California Press, 1995, p. 31-51, ici p. 36, 40, ainsi que les articles de P. Sloan, D. Carrithers et G. Hatfield dans le même volume.

6 M. Crépon distingue «l'enquête anthropologique » menée par Buffon et Voltaire et le « tableau historique » de Montesquieu (Les Géographies de l'esprit, Paris, Payot, 1996, chap. 1 et 2).

7 P. Manent, La Cité de l'homme, Paris, Champs Flammarion, 1994, chap. 2, ici p. 79.

8 L. Althusser, Montesquieu. La politique et l'histoire, Paris, P.U.F., 1959, p. 15.

9 Mes Pensées, $\mathrm{n}^{\circ}$ 1940. Sur la «science nouvelle» découverte par Montesquieu, voir B. Binoche, Introduction à «De l'esprit des lois» de Montesquieu, Paris, P.U.F., 1998.

10 EL, XIX, 4. Voir F. Markovits, «Montesquieu : l'esprit d'un peuple. Une histoire expérimentale », in Former un nouveau peuple?, J. Boulad-Ayoub éd., Presses Universitaires de Laval, L'Harmattan, 1996, p. 207-236. 


\section{L'Esprit des lois : une relégation de l'anthropologie?}

Dans la Préface de L'Esprit des lois, Montesquieu accompagne la présentation de son dessein d'une phrase énigmatique : "L'homme, cet être flexible, se pliant dans la société aux pensées et aux impressions des autres, est également capable de connaître sa propre nature lorsqu'on la lui montre, et d'en perdre jusqu'au sentiment lorsqu'on la lui dérobe ». Quelle est donc cette «nature » occultée que L'Esprit des lois prétend révéler à l'homme social et faire accéder ainsi à la conscience de soi ? Le livre I semble destiné à y répondre, en envisageant la place de l'homme au sein de la légalité naturelle, entre le monde physique des corps (régi par des lois nécessaires du mouvement) et le monde moral des intelligences (régi par des lois invariables de justice, par des rapports moraux plutôt que par des rapports physiques)11. Or selon Montesquieu, «il s'en faut bien que le monde intelligent soit aussi bien gouverné que le monde physique » (I, 1).

La question «qu'est-ce que l'homme?» acquiert ici une dimension nouvelle. En tant qu'être mixte, corps et intelligence c'est-à-dire sensibilité, l'homme est dans l'entre-deux, ce pourquoi il ne suit pas nécessairement les lois de sa nature. Au livre I de L'Esprit des lois ${ }^{12}$, la place de l'homme se trouve définie comme une non-place, dans un déplacement de la double tradition de l'échelle des êtres et de la dignitas hominis : les qualités « spirituelles » de l'homme (raison, liberté) ne l'élèvent pas au sommet de la création, bien au contraire. Montesquieu soutient deux thèses conjointes : si «l'homme a ses lois », il est par ailleurs l'être le moins assujetti à ses lois qui soit ${ }^{13}$. Un mauvais usage des passions comme de la raison peut le conduire à méconnaître ou à transgresser les lois d'équité qui sont les rapports moraux qu'il doit suivre: "Les faibles connaissances qu'il a, il les perd encore : comme créature sensible il devient sujet à mille passions ». La sensibilité humaine est plus instable que celle des animaux qui «ne font pas un aussi mauvais usage de leurs passions » (I, 1). Telle est la raison pour laquelle l'homme doit être lié à l'ordre des devoirs par d'autres lois (religieuses, morales et surtout politiques).

Dès lors, il faut s'entendre sur les «lois de la nature » humaine invoquées dans la fiction de l'état de nature. Le tableau de l'homme naturel comme être timide, craintif et pacifique s'inscrit dans un dispositif polémique: partageant avec Pufendorf, contempteur de Hobbes, la vision pacifique de l'état de nature, Montesquieu ne souscrit pas pour autant à sa théorie de l'homme craintif et raisonnable ${ }^{14}$. Avant le second Discours, L'Esprit des lois fait du sauvage un animal borné, en deçà du bien et du mal, incapable d'accéder à la loi naturelle. La distinction est nette entre les rapports d'équité concernant l'être «intelligent» et les «lois de la nature » qui dérivent de l'être sensible de l'homme: les lois découvertes à l'état de nature - désir de paix, de nourriture, de partenaires sexuels et sociaux afin de vivre ensemble - sont d'abord issues du désir de conservation, antérieurement au développement de la raison.

Pour autant, il ne s'agit pas non plus de figer «l'homme naturel» en l'apparentant à un être primitif, sauvage et quasi-animal. Dès l'état de nature s'introduit une évolution historique, une émancipation à l'égard de l'animalité à mesure que l'homme acquiert des idées et que sa sensibilité, en conséquence, s'éduque ou se cultive. Grâce à l'attraction des deux sexes et à la « prière naturelle » qu'ils se font, la socialisation s'amorce à partir d'un besoin primaire. C'est cette socialisation qui se poursuit ensuite à une plus vaste échelle : si l'être humain est d'emblée un

11 Voir B. Binoche, Introduction à «De l'esprit des lois» de Montesquieu, Paris, P.U.F., 1998, p. 31-44 ; C. Spector, "Quelle justice? Quelle rationalité ? La mesure du droit dans L'Esprit des lois», dans Montesquieu en 2005, C. Volpilhac-Auger éd., Oxford, Voltaire Foundation, 2005, p. 219-242.

${ }^{12}$ Ce livre, mis au net par le secrétaire « $\mathrm{H}$ » dans le Manuscrit BNF dont nous disposons (actif auprès de Montesquieu en 1741-1742), a été rajouté après coup, ainsi que le livre II. Le livre III devait à l'origine constituer l'ouverture de L'Esprit des lois (voir C. Volpilhac-Auger, in Oeuvres complètes de Montesquieu, t. III, Oxford, Voltaire Foundation, 2008, p. 3).

13 Voir J.-P. Courtois, Inflexions de la rationalité dans "L'Esprit des lois», Paris, P.U.F., 1999, p. 192-199; C. Spector, Montesquieu. Liberté, droit et histoire, Paris, Michalon, 2010, chap. 1.

14 Voir Pufendorf, Le Droit de la nature et des gens, trad. J. Barbeyrac, Amsterdam 1734, II, I, \ 9. Sur le rapport à Hobbes, voir le manuscrit (OC, t. III, p. 9). 
animal social, sa sensibilité au plaisir se raffine par les connaissances qu'il acquiert. Aussi peut-il entrer dans le champ de la culture, qui est celui de la vie sociable. Il n'est pas exclu d'invoquer le concept de "perfectibilité », malgré l'absence littérale du terme ${ }^{15}$ : à l'origine, l'homme ne dispose que qu'une faculté de connaitre, sans idées innées ni dispositions morales. Seules les notions simples associées à la subsistance sont à sa portée, avant qu'un processus d'éducation ne le conduise à acquérir des idées complexes et spéculatives ${ }^{16}$. L'homme est cet être dont la nature n'est qu'une capacité d'altération: à l'état de nature, il est certes pourvu de lois qui «dérivent uniquement de la constitution de [son] être ", mais il s'agit de sentiments voués à l'évolution - la genèse des passions suivant celle des idées, comme le montrera à son tour Rousseau. L'homme n'a pas réellement de "constitution»: ses désirs, en société, se pluralisent selon la nature des sociétés et des régimes politiques, en fonction des idées nouvelles dont l'homme désormais dispose.

A ce titre, la nature de l'homme n'est rien d'autre que désir et activité ou force, vouée à se modifier selon le milieu où elle s'exerce. Le livre I de L'Esprit des lois met en scène une dynamique sociale : l'entrée en société suscite une métamorphose des croyances et des passions. Dans l'état de nature, le sentiment de faiblesse associé à la crainte réciproque entre les individus suscitait la fuite (les faiblesses égales se repoussent), puis l'union (elles s'attirent). Or de cette union émerge un sentiment de force qui provoque à son tour la désunion, voire la guerre : "Sitôt que les hommes sont en société, ils perdent le sentiment de leur faiblesse ; l'égalité, qui était entre eux, cesse, et l'état de guerre commence. Chaque société particulière vient à sentir sa force ; ce qui produit un état de guerre de nation à nation. Les particuliers, dans chaque société, commencent à sentir leur force ; ils cherchent à tourner en leur faveur les principaux avantages de cette société ; ce qui fait entre eux un état de guerre » $(\mathrm{I}, 3)$. C'est à cet état de guerre que remédie l'institution de l'Etat, qui procède de l'union des « forces » et des « volontés » qui les dirigent. S'opère alors en l'homme une modification associée à la socialisation du désir, désir qui devient " passion » en se différenciant selon les sociétés et selon les régimes. La question de l'homme devient ainsi celle des hommes et de leurs passions, qui sont leurs véritables ressorts.

Il faut donc assigner sa juste place au concept de «nature humaine » dans L'Esprit des lois. A l'issue du livre I, l'anthropologie se pluralise : alors que les corps sont mus selon les lois du mouvement, les hommes sont mus par des passions orientées selon certaines croyances ; l'action des institutions sur ces croyances donne lieu à une formation singulière des passions dans les différents régimes et les différentes nations. L'impact des « lois de la nature » dans l'état civil est néanmoins conservé : en faisant de la défense naturelle et de la pudeur naturelle des lois universelles ${ }^{17}$, Montesquieu maintient une réalité intangible - une sensibilité vulnérable à la cruauté associée aux trois formes de servitude (domestique, civile et politique). Contrairement à ce que prétendent Leo Strauss et ses disciples ${ }^{18}$, le concept de «nature humaine » ne disparait pas de L'Esprit des lois : la nature humaine est invoquée négativement, contre les abus de pouvoir. S'il met en crise le droit naturel moderne, Montesquieu esquisse les contours d'une anthropologie de la vulnérabilité - faiblesse de la chair face à la force pure - qui permet de condamner le despotisme, régime le plus contrenature qui soit : «le despotisme cause à la nature humaine des maux effroyables »; les despotes «se jouent de la nature humaine », l'insultent. Montesquieu n'use jamais plus du concept de « nature humaine » que lorsqu'il l'envisage négativement, face à la cruauté despotique : «Lorsque nous lisons, dans les histoires, les exemples de la justice atroce des sultans, nous sentons avec une espèce de douleur les maux de la nature humaine $»^{19}$.

15 L'idée, selon nous est présente dès l'Essai sur les causes (OC, IX, p. 246-249). Sur l'évolution du concept, voir L'bomme perfectible, B. Binoche éd., Seyssel, Champ Vallon, 2004.

$16 L^{\prime} E$ ssai sur les causes décrit ce processus d'éducation, qui est un processus de socialisation.

17 EL, X, 2 ; XV, 12 ; XVI, 2 ; XXVI, 3.

${ }^{18}$ Les cours de Leo Strauss à l'Université de Chicago en 1965 et 1966, inédits à ce jour, nous ont été aimablement communiqués par le directeur du Leo Strauss Center, Nathan Tarkov, que nous souhaitons remercier ici. Voir C. Spector, «Montesquieu et la crise du droit naturel moderne. L'exégèse straussienne », numéro spécial sur «Montesquieu », D. de Casabianca dir., 2013 (1), p. 65-78.

${ }^{19}$ EL, II, 4; VII, 9 ; VIII, 8 ; VI, 9. 


\section{Deux anthropologies distinctes?}

\section{1) La théorie des principes comme théorie des forces}

En tant que théorie de la force qui dynamise la sensibilité, une réflexion sur la nature des peuples se déploie tout au long de L'Esprit des lois. A la suite du livre I, Montesquieu élabore deux anthropologies concurrentes: une anthropologie politique, associée à la théorie des principes, passions politiques dominantes dans les différents Etats ; une anthropologie culturelle, qui explique la diversité des croyances et des passions, des esprits et des caractères, au niveau des nations. La difficulté, bien connue des exégètes, est dès lors d'articuler ces deux moments, la théorie des «types » et celle des « causes $»^{20}$ - ce qui s'opère, non sans tensions, dans la réflexion consacrée à l'« esprit général » des peuples.

Dans les premiers livres de L'Esprit des lois, l'anthropologie se déploie d'abord comme théorie des principes, qui sont les «ressorts » qui font mouvoir les hommes au sein de chaque régime : « la force du principe entraîne tout »(VIII, 11). A l'issue du livre I, la cupidité et l'ambition sont apparues comme les passions sociales dominantes de l'homme, celles qui, procédant du sentiment de sa force et s'associant aux idées d'empire et de domination, visent les «avantages sociaux ». Dans l'état civil primitif, la force individuelle s'exerce pour surmonter celle des autres, grâce à l'acquisition des biens disponibles (richesses, honneurs, pouvoirs). Or comme des forces, la cupidité et l'ambition peuvent connaître différents destins : elles peuvent être soit réorientées du particulier vers le public (théorie de la vertu); soit exprimées et régulées (théorie de l'honneur); soit inhibées (théorie de la crainte) $)^{21}$.

En démocratie, les passions associées au désir de distinction ou de domination sont réorientées vers l'intérêt public. La passion change d'objet : l'amour de soi devient amour de la patrie et amour des lois, amour de l'égalité et de la frugalité. L'image célèbre du monastère en témoigne: «Moins nous pouvons satisfaire nos passions particulières, plus nous nous livrons aux générales. Pourquoi les moines aiment-ils tant leur ordre? C'est justement par l'endroit qui fait qu'il leur est insupportable. Leur règle les prive de toutes les choses sur lesquelles les passions ordinaires s'appuient : reste donc cette passion pour la règle même qui les afflige. Plus elle est austère, c'est-à-dire, plus elle retranche de leurs penchants, plus elle donne de force à ceux qu'elle leur laisse » $(\mathrm{V}, 2)$. La vertu politique est une force d'âme propre à soutenir la démocratie ou, si elle s'affaiblit, à la corrompre: «Les politiques grecs, qui vivaient dans le gouvernement populaire, ne reconnaissaient d'autre force qui pût les soutenir que celle de la vertu. [...] Lorsque cette vertu cesse, l'ambition entre dans les cœurs qui peuvent la recevoir, et l'avarice entre dans tous. Les désirs changent d'objets : ce qu'on aimait, on ne l'aime plus... » (III, 3). Dans la Rome décadente, le peuple «perdit la force de la liberté pour tomber dans la faiblesse de la licence » (VIII, 12).

Ce vocabulaire de la force ne concerne pas seulement la vertu comme force d'âme. On le retrouve à propos de l'honneur monarchique, puisque les forces de l'ambition sont ici exprimées et régulées, mais non inhibées. D’un côté, la machine monarchique économise la force de la vertu: «dans les monarchies, la politique fait faire les grandes choses avec le moins de vertu

20 On ne reviendra pas ici sur cette question classique, aussi bien du point de vue de L. Althusser, qui évoque l'équivoque du concept de principe et du concept de mœurs (op. cit., p. 62), que de R. Aron, qui souligne la tension entre théorie des causes et théorie des types (R. Aron, Les Etapes de la pensée sociologique, Paris, Gallimard, 1967, chap. 1). Voir G. Benrekassa, Montesquieu : la liberté et l'bistoire, Paris, Librairie générale française, 1987, chap. 5.

21 EL, IV, 4-5 ; V, 2-3; EL, III, 7; IV, 2 ; EL, IV, 3. 
qu'elle peut; comme, dans les plus belles machines, l'art emploie aussi peu de mouvements, de forces et de roues qu'il est possible» (III, 5). De l'autre, elle bénéficie des forces dirigées par l'ambition, que les lois peuvent au besoin réprimer: "L'ambition est pernicieuse dans une république. Elle a de bons effets dans la monarchie; elle donne la vie à ce gouvernement; et on y a cet avantage, qu'elle n'y est pas dangereuse, parce qu'elle y peut être sans cesse réprimée. Vous diriez qu'il en est comme du système de l'univers, où il y a une force qui éloigne sans cesse du centre tous les corps, et une force de pesanteur qui les y ramène. L'honneur fait mouvoir toutes les parties du corps politique ; il les lie par son action même; et il se trouve que chacun va au bien commun, croyant aller à ses intérêts particuliers $»^{22}$. Quand bien même l'homme d'honneur serait régi par un principe " faux », de l'ordre du préjugé, son attrait pour les honneurs le conduit à son insu au bien public. La force des individus, dirigée par des croyances singulières, se trouve ainsi mobilisée au profit de l'Etat: «Et n'est-ce pas beaucoup d'obliger les hommes à faire toutes les actions difficiles, et qui demandent de la force, sans autre récompense que le bruit de ces actions ? $\gg .^{23}$

Enfin, le despotisme ne se conserve qu'à condition d'inhiber toutes les forces morales disponibles, pour mieux faire dominer celle - purement physique - du despote. Autant que la vertu, l'honneur est absent du despotisme : «Il fait gloire de mépriser la vie, et le despote n'a de force que parce qu'il peut l'ôter $»^{24}$. L'économie passionnelle y est extrêmement simple, puisque le pouvoir, ici, se réduit à l'exercice de la domination ou à l'attrait des récompenses matérielles, anéantissant toute noble ambition et toute force d'âme (III, 9). La crainte despotique prolonge le sentiment animal instinctif: envers de l'instinct de conservation, elle n'inclut aucun rapport immanent à la loi - contrairement à l'amour des lois républicain ou au respect du code de l'honneur monarchique. Aussi le despote ne maintient-il sa force qu'en inhibant l'aptitude à délibérer et à vouloir, la prudence et le courage qui constituent l'humanité socialisée (IV, 3).

Par là même, Montesquieu transpose l'art politique aristotélicien dans le champ de la physique newtonienne. La théorie des principes s'appuie sur une mécanique des forces. Dans un régime donné, le rapport entre nature et principe se comprend selon le schème de l'actionréaction: "Ce rapport des lois avec ce principe tend tous les ressorts du gouvernement; et ce principe en reçoit à son tour une nouvelle force. C'est ainsi que, dans les mouvements physiques, l'action est toujours suivie d'une réaction» $(\mathrm{V}, 1)$. Dans chaque espèce de gouvernement, l'éducation à la vie sociale est formation des croyances et des passions - le ressort de la machine humaine étant en même temps le ressort qui permet au gouvernement de se mouvoir et de se conserver. Corrélativement, la modération (vertu suprême du législateur) est un savoir de l'équilibre des forces ou plutôt des puissances : «Pour former un gouvernement modéré, il faut combiner les puissances, les régler, les tempérer, les faire agir ; donner, pour ainsi dire, un lest à l'une, pour la mettre en état de résister à une autre ; c'est un chef-d'œuvre de législation, que le hasard fait rarement, et que rarement on laisse faire à la prudence » $(\mathrm{V}, 14)$. Lorsqu'elle n'est pas inhibée par la crainte ou réorientée par la vertu, la passion du pouvoir s'exprime inexorablement :

Lorsque, dans un siècle, ou dans un gouvernement, on voit les divers corps de l'État chercher à augmenter leur autorité, et à prendre les uns sur les autres de certains avantages, on se tromperait souvent si l'on regardait leurs entreprises comme une marque certaine de leur corruption. Par un malheur attaché à la condition humaine, les grands hommes modérés sont rares; et, comme il est toujours plus aisé de suivre sa force que de l'arrêter, peut-être, dans la classe des gens supérieurs, est-il plus facile de trouver des gens extrêmement vertueux, que des hommes extrêmement sages ${ }^{25}$.

Pour autant, la modération (hormis le cas singulier de l'aristocratie) n'est pas conçue comme une qualité de l'âme: le désir de pouvoir doit trouver des forces qui lui résistent. L'expression célèbre selon laquelle « pour qu'on ne puisse abuser du pouvoir, il faut que, par la

22 EL, III, 7. Pour l'explication de ce mécanisme, nous nous permettons de renvoyer à notre Montesquieu. Pouvoirs, richesses et sociétés, Paris, P.U.F., 2004 (rééd. Paris, Hermann, 2011), chap. 1.

${ }^{23}$ Ibid., n. s.

24 EL, III, 8 ; voir LP, 89-90 et notre Montesquieu. Les «Lettres persanes », Paris, P.U.F., 1997.

25 EL, XXVIII, 41, n. s. 
disposition des choses, le pouvoir arrête le pouvoir » (XI, 4) trouve ici son fondement : l'homme civil, fût-il vertueux, a besoin de limites.

A cet égard, le savoir spéculatif est toujours au service d'un intérêt pratique. Le projet de Montesquieu n'est pas d'élaborer une physique politique, mais de réfléchir au bon usage des lois dans leurs rapports aux circonstances : le législateur doit savoir comment corriger le droit ou agir opportunément sur les pratiques ${ }^{26}$. L'Esprit des lois n'a d'autre objet que d'identifier les conditions de la liberté politique et civile, et éclairer le législateur sur les normes qui conviennent à des hommes libres, en situation.

\section{2) La théorie des fibres comme théorie des forces}

Cependant, cette anthropologie politique n'est pas seule en lice dans L'Esprit des lois. Dans la troisième partie, l'anthropologie s'aborde sous l'angle d'une théorie des causes, comme théorie des rapports du physique et du moral associée à l'influence des climats (livre XIV à XVII). C'est ici la fibre qui compose l'être humain, et son système musculaire et nerveux qui fait figure de force. Comme l'a montré Denis de Casabianca, la caractéristique première de la fibre est selon Montesquieu sa contractilité, qui détermine sa qualité : plus resserrée, la fibre a plus de force et de « ressort ». Dans le sillage de Baglivi, Boerhaave, Glisson ou Arbuthnot, le livre XIV fait droit à une conception médicale mécaniste, confortée par l'usage du microscope ${ }^{27}$. En fonction des milieux, la machine humaine est vouée à l'activité (force, vigueur, courage) ou au contraire à la passivité (faiblesse, paresse, lâcheté). La puissance ou l'impuissance d'agir se communique de l'esprit au corps et du corps à l'esprit par les fibres nerveuses qui convergent dans le cerveau (l'araignée au centre de sa toile ${ }^{28}$ ). Lorsque la chaleur du climat devient excessive, la paresse du corps devient abattement de l'esprit et découragement du vouloir, suscitant la servitude civile et politique: "Aucune curiosité, aucune noble entreprise, aucun sentiment généreux; les inclinations y seront toutes passives; la paresse y fera le bonheur; la plupart des châtiments y seront moins difficiles à soutenir que l'action de l'âme, et la servitude moins insupportable que la force d'esprit qui est nécessaire pour se conduire soi-même » (XIV, 3).

Sans doute Pierre Bourdieu n'a-t-il pas tort d'ironiser sur une «rhétorique de la scientificité » dont les effets idéologiques sont douteux ${ }^{29}$. Il reste qu'un paradigme médical de l'unité de la sensibilité se substitue ici à une métaphysique de l'union des substances. Montesquieu observe les effets produits par le milieu sur l'économie animale afin de découvrir les conditions d'émergence d'une sensibilité collective active ou passive. Le véritable dessein de L'Esprit des lois est d'en déterminer les effets sur la puissance (liée, chez les modernes, à l'activité économique) et sur la liberté (liée à l'activité militaire et politique). Le concept de force noue ainsi anthropologie et politique: il n'existe pas de solution de continuité entre le ressort des fibres et celui des gouvernements. C'est du sentiment de sa force, prévalent au Nord, que procèdent le sentiment de courage, de confiance en soi, d'aptitude à conquérir ou à défendre sa liberté : vigueur active qui passe du corps à l'esprit de même que, dans les contrées torrides, le sentiment de faiblesse passe du

${ }^{26}$ Voir D. de Casabianca, L’Esprit des lois. Montesquieu, Ellipses, « Philo-œuvres », 2003.

27 Nous ne pouvons entrer ici dans le détail de l'analyse. Voir le travail remarquable de D. de Casabianca, Montesquieu. De l'étude des sciences à l'esprit des lois, Paris, Honoré Champion, 2008, et celui de G. Barrera dans son annotation de l'Essai sur les causes (in CEuvres et écrits divers II, sous la direction de P. Rétat, OC, t. IX, Oxford, Voltaire Foundation, 2006, p. 205-270). Selon ce dernier, dans les leçons de «physiologie cérébrale et générale » qui forment la première partie de l'Essai sur les causes, Montesquieu, tout en s'appuyant sur Malebranche, en complète et en infléchit les données au regard de la science médicale la plus actuelle vers 1730. G. Barrera souligne, au nombre des sources les plus importantes de cet opuscule, les traités Des maladies ou Institutions de médecine de Boerhaave, les Tables anatomiques d'Eustache, la Neurographie universelle de Vieussens, les Essais de physique de Sénac, les Euvres complètes de Malpighi, ou encore les Opuscules de Stahl. Mais la théorie des fibres est celle des plus grands médecins du temps de Montesquieu (Borelli, Willis, Glisson, Boerhaave et Winslow). Un recueil d'extraits de Montesquieu intitulé Anatomica est aujourd'hui perdu.

28 Voir Causes, op. cit., p. 240

29 P. Bourdieu, "La rhétorique de la scientificité : contribution à une analyse de l'effet Montesquieu », in Ce que parler veut dire, Paris, Fayard, 1982, p. 227-239. 
corps à l'esprit, les inclinations paresseuses et passives suscitant censément l'accoutumance à la servitude.

\section{Charles Bonnet ou la naturalisation de l'anthropologie}

Faut-il dès lors faire de Montesquieu l'un des fondateurs méconnus de l'anthropologie ? L'auteur de L'Esprit des lois aurait-il tenté d'accomplir le projet d'Auguste Comte lorsque celui-ci entendait appuyer la sociologie sur la biologie et la physiologie cérébrale - même si Comte regrettait que Montesquieu n'ait pu connaittre la "physiologie biologique » de Haller, Jussieu, Linné, Buffon, et Vicq d'Azyr ${ }^{30}$ ? La réponse est négative : Montesquieu ne partage pas le projet d'une anthropologie naturaliste. Certes, son œuvre aborde des questions relatives aux rapports du physique et du moral ou à la place de l'homme dans le règne animal31. Sans pouvoir faire droit ici à l'ensemble des opuscules et des œuvres ${ }^{32}$, on soulignera la trace d'un intérêt précoce pour la question de l'union de l'âme et du corps : la «Resomption de l'observation de Monsieur Doazan [médecin, vers 1677-1745] sur un enfant né sans cerveau » (1718) concerne les rapports entre la pensée et le cerveau, dans un débat autour de Malebranche ${ }^{33}$. Un enfant pourrait-il vivre sans cerveau, comme l'affirme l'auteur? Sans prendre position devant l'Académie, le rapporteur expose les effets de cette observation anatomique sur la philosophie :

De pareilles observations derangent plus de sistêmes que les philosophes n'en sauroient faire en un siècle. Le Pere Malebranche a seché trente ans pour nous faire comprendre l'harmonie des idées, et des traces qui produisent l'imagination et la memoire : mais ce beau systême tombe en ruine devant votre observation. Il faut recommencer a expliquer tout ce qui a du raport a l'union de l'ame et du corps, et aux facultés relatives de ces deux substances. Il faut regarder le cerveau comme une vile glande uniquement occupée à separer quelques serosités. Cependant, Monsieur, voyés quelles gens ce sont que les philosophes; quoique vous veniés ravager nos terres, vous nous voyés tous prêts a vous remercier, et a vous rendres graces du desordre même que vous y faites ${ }^{34}$.

Mais cette critique du malebranchisme ne donne pas lieu à l'édification d'un système concurrent. Dans l'article «Goût » qu'il destine à L'Encyclopédie, Montesquieu est au plus proche d'une telle tentative, ordonnée à un savoir pratique des «plaisirs de l'âme ». Là encore, l'auteur rompt avec tout dualisme substantiel ; la dualité de l'âme et du corps est fonctionnelle ; connaitre et sentir ne sont qu'une seule et même chose : «car, quoique nous opposions l'idée au sentiment, cependant lorsqu'elle [l'âme] voit une chose, elle la sent $»^{35}$. La nature des plaisirs éprouvés s'explique par des raisons physiologiques, liées à l'unité de la sensibilité : «l'âme ne peut pas soutenir longtemps les mêmes situations, parce qu'elle est liée à un corps qui ne peut les souffrir ; pour que notre âme soit excitée, il faut que les esprits coulent dans les nerfs $»^{36}$. Il ne s'agit donc

30 Comte, Cours de philosophie positive, «La physique sociale », Paris, Hermann, 1975, leçon n 47, p. 89.

31 Montesquieu consacre plusieurs réflexions, plus ou moins ironiques, à la place de l'homme. Certaines sont savoureuses: «Melon disait agréablement que l'homme est un singe manqué. Cela a un sens dans le rapport que les hommes ont des imperfections que les bêtes n'ont point» (MP, 1440) ; "Si l'espèce humaine périssait, quelle différence dans les autres espèces? Comment les poules, les brebis, pourraient-elles se soutenir? » (MP, 2016) ; «Les animaux ont plus d'esprit à mesure qu'ils ont plus de facilité pour l'exercer: les singes, avec leurs mains; les éléphants, avec leur trompe, les castors, avec leur queue ; les hommes, avec leurs bras et leur langue » (MP, 788).

32 Nous nous contenterons de renvoyer à notre article «Âme » et « Esprit, Esprit général » dans le Dictionnaire Montesquieu (http://Dictionnaire-Montesquieu.ens-lsh.fr) et surtout au remarquable livre de D. de Casabianca, Montesquieu. De l'étude des sciences à l'esprit des lois, op. cit.

33 Le débat met aussi en jeu Fontenelle, pour qui le problème de l'union reste insoluble (Histoire de l'Académie royale des sciences, cité dans L'Idée de nature en France dans la première moitié du XVIII siècle, rééd. Paris, Albin Michel, 1994, p. 679).

34 CEuvres et Ecrits divers, I, OC, t. VIII, sous la direction de P. Rétat, Oxford, Voltaire Foundation, 2003, p. 172. Par ailleurs, Montesquieu s'est mis par ailleurs au nombre des «cartésiens rigides » contre les malebranchistes à propos de la production mécaniste du végétal : voir l'Essai d'observations sur l'histoire naturelle, même volume, p. 213.

35 Essai sur le goût, in CEuvres complètes de Montesquieu, Oxford, Voltaire Foundation, t. IX, 2006, p. 490. Sur la datation de cet essai et ses diverses strates, voir l'introduction de Annie Beck. Voir aussi C. Spector, "Une théorie matérialiste du goût peut-elle produire l'évaluation esthétique? Montesquieu, de L'Esprit des lois à L'Essai sur le goût», Corpus, n 40, 2002, p. 167-213.

36 Ibid., p. 497. 
pas de nier la dualité de l'âme et du corps, mais de penser leurs rapports fonctionnels. Montesquieu y insistait déjà dans un fragment de sa dissertation sur la « différence des esprits » :

Lorsque les médecins et les auteurs moraux traitent des passions, ils ne parlent jamais la même langue : les moraux mettent trop sur le compte de l'âme ; les autres, trop sur celui du corps ; les uns regardent l'homme comme un esprit; les autres, plus comme la machine d'un artisan. Mais l'homme est également composé de deux substances, qui, chacune, comme par un flux et reflux, exercent et souffrent l'empire37.

Peu importe, à cet égard, que les rapports qui unissent l'âme au corps échappent à la prise rationnelle. Ce qui est essentiel est que l'esprit se structure dans l'activité de mise en rapport qui l'ancre dans le sensible: l'âme n'est que le centre topique des rapports qui constituent sa machine; les rapports qui structurent l'esprit s'enracinent dans la configuration active de la sensibilités8.

Pourtant, L'Esprit des lois, qui prend la suite de la tentative avortée de L'Essai sur les causes qui peuvent affecter les esprits et les caractères, n'élabore pas ce savoir anthropologique. La confrontation avec Charles Bonnet, grand lecteur de L'Esprit des lois qui crédita son auteur d'être le « Newton du monde moral $\aleph^{39}$, est révélatrice. Souvent mentionné au rang des œuvres fondatrices de l'anthropologie, L'Essai de psychologie l'affirme : l'ignorance où nous sommes de la nature de l'âme et de son union avec le corps ${ }^{40}$ n'empêche pas de dire que l'homme est un être mixte, composé de deux substances, spirituelle et corporelle ${ }^{41}$. Utilisant la métaphore de l'âme claveciniste, Bonnet élabore une théorie des fibres nerveuses qui préside à l'organisation du cerveau et à l'émergence de la sensibilité :

Le siège de l'âme est une petite Machine prodigieusement composée, et pourtant fort simple dans sa composition. C'est un abrégé très complet de tout le genre nerveux, une neurologie en miniature. On peut se représenter et admirable instrument des Opérations de notre âme, sous l'image d'un clavecin, d'une Orgue, ou d'une Horloge [...]. L'âme est le musicien qui exécute sur cette machine différents airs, ou qui juge de ceux qui sont exécutés, et qui les répète. Chaque fibre est une espèce de touche, ou de marteau destiné à rendre un certain ton ${ }^{42}$.

L'Essai analytique sur les facultés de l'âme étend la méthode de l'histoire naturelle à la psychologie individuelle des hommes. S'il est vain de vouloir connaitre la nature des choses, il faut en revanche observer la mécanique de nos facultés et substituer la physique (l'étude du jeu des fibres) à la métaphysique ${ }^{43}$. L'ignorance du «secret» de l'union des substances et de leur " essence réelle » n'empêche pas toute connaissance de l'homme44 ni toute "science de l'âme », selon la méthode expérimentale: suivant la méthode newtonienne, l'ignorance des causes ne contrevient pas à l'étude des effets ni à la découverte des lois ${ }^{45}$.

Ainsi se trouve redéfinie la question classique - qu'est-ce que l'homme ${ }^{46}$ ? Les « réflexions générales et préliminaires sur la nature de l'homme » de Bonnet le conduisent à une théorie des lois

37 MP, 2035 (Academica). Dans les Lettres persanes, Usbek se demande s'il ne faut pas «traiter l'homme comme sensible au lieu de le traiter comme raisonnable» sachant que "l'âme, unie avec le corps, en est sans cesse tyrannisée » (LP, $31[33])$.

38 MP, 157. Voir C. Spector, «Montesquieu et la métaphysique dans les Pensées », Revue Montesquieu, n ${ }^{7}, 2003-2004$, p. 113-134.

39 «Newton a découvert les lois du monde matériel, vous avez découvert, Monsieur, les lois du monde intellectuel » (Charles Bonnet, lettre à Montesquieu du 14 novembre 1753, in OC, t. III, p. 1478).

40 Bonnet, Essai de psychologie, on considérations sur les opérations de l'âme, sur l'habitude et sur l'éducation, Hildesheim, New York, Georg Olms Verlag, 1978 (fac simile, Londres, 1755), p. 1-2 (orth. modernisée).

41 Ibid., p. 3.

42 Ibid., p. 13.

43 Bonnet, Essai analytique sur les facultés de l'âme, Hilesheim, New York, Georg Olms Verlag, 1973 (Copenhague, 1760), p. XIII.

44 Ibid., p. XIV.

45 Ibid., p. XVI-XVII.

46 Ibid., p. 1. 
de l'union, rapports constants entre phénomènes variables ${ }^{47}$. La théorie des fibres nerveuses et de l'émergence de la sensibilité explique les rapports du physique et du moral. L'organisation du cerveau est déterminante :

L'anatomie nous découvre dans les nerfs un des principaux instruments de l'Union. Cette science aujourd'hui si perfectionnée, nous démontre que l'âme ne sent et ne peut sentir qu'à l'aide des nerfs. Elle prouve que les nerfs tirent leur origine du Cerveau, et que delà ils se répandent dans tous les réduits du corps. La découverte de l'origine des nerfs, a conduit à placer l'âme dans le cerveau ${ }^{48}$.

Doit-on en conclure que Bonnet accomplit ce que Montesquieu avait entrepris, et que son œuvre contient, en quelque sorte, l'anthropologie sous-jacente à L'Esprit des lois ? A la fin de son ouvrage, un chapitre intitulé Observations sur quelques endroits de "L'Esprit des lois» permet d'esquisser une réponse. Après avoir dit toute son admiration pour le " génie » de Montesquieu", Bonnet critique en effet le livre I de L'Esprit des lois. Outre la définition liminaire de la loi, qui donne lieu à une correspondance entre les deux auteurs ${ }^{50}$, le naturaliste récuse la différence introduite entre le gouvernement du monde intelligent et celui du monde physique :

Chaque être intelligent a des lois invariables de leur nature : ces lois sont celles de sa nature particulière. $S a$ nature est ses idées, ses penchants, ses affections, en un mot tout ce qui constitue son caractère individuel. Son caractère fait son essence morale ou intellectuelle; car ce n'est pas la simple capacité de connaître qui forme cette essence. Un être n'est pas intelligent, simplement parce qu'il a la capacité de l'être : il est intelligent parce qu'il a des notions, et il ne peut agir qu'en conséquence de ce qu'il connait.

L'assemblage des lois qui meuvent les êtres particuliers intelligents, forme donc le Système général des lois qui gouvernent le monde intelligent.

Le monde intelligent est donc gouverné par des lois invariables ; car il n'est point d'être intelligent qui n'agisse d'une manière conforme à son essence intellectuelle, ou aux idées qu'il se fait des choses.

Le monde intelligent est donc aussi bien gouverné que le monde physique, puisque les êtres particuliers intelligents sont aussi fidèles à suivre les lois de leur nature individuelle, que les corps le sont à suivre les lois de la leur ${ }^{51}$.

Selon Bonnet, Montesquieu a eu tort de différencier le sort des hommes de celui des animaux ou des plantes. Toute la nature suit ses lois de la même façon: l'homme suit donc les lois de sa nature intelligente. Apparait ici la différence majeure avec Montesquieu qui s'en tient, pour sa part, à une psychologie collective, car les causes apparaissent de façon moins arbitraire au niveau des grands nombres ${ }^{52}$.

Si Montesquieu fait partie des théoriciens qui assument un rôle fondateur dans l'anthropologie, c'est donc au sens où son histoire naturelle des institutions suppose une histoire naturelle des hommes. Dans L'Esprit des lois du moins, son anthropologie reste du côté des «variétés de l'espèce humaine » et non des lois universelles de la sensibilité. En ce sens, Rousseau avait vu juste en plaçant Montesquieu au nombre des philosophes qui pourraient contribuer à la connaissance empirique du genre humain plus qu'à une réflexion universelle sur les " principes » du droit politique ${ }^{53}$.

Il reste que les questions anthropologiques, dans L'Esprit des lois, ne sont pas abordées pour elles-mêmes. Elles ne valent que dans la mesure où elles peuvent constituer un savoir utile aux peuples et aux législateurs : les dispositions d'esprit des nations permettent de concevoir avec

47 «Je conçois donc l'Union de l'âme et du corps et leur influence réciproque, comme un phénomène dont j'étudie les lois, et dont je fais profession d'ignorer profondément le comment» (ibid., p. 5).

48 Ibid., p. 18.

49 Ibid., p. 541.

50 Sur cette controverse, voir A. Postigliola, «Montesquieu e Bonnet : la controversia sul concetto di legge », in La politica della ragione, P. Casini éd., Bologne, Il Mulino, 1978, p. 43-69.

51 Bonnet, Essai analytique sur les facultés de l'âme, op. cit., p. 544-545.

52 Voir Causes, p. 219.

53 Rousseau, Discours sur les origines et les fondements de l'inégalité parmi les hommes, note X, in OC, t. III, Paris, Gallimard, 1964, p. 213-214 ; Emile, in OC, t. IV, Paris, Gallimard, 1969, p. 836. 
prudence les dispositions des lois. Faut-il en conclure que l'étude en situation de «l'être flexible» qu'est l'homme conduit à fonder la science sociale ou la science politique ${ }^{54}$ ? Du moins ces savoirs relèvent-ils d'une matrice commune. A cet égard, la place de L'Esprit des lois dans «l'anthropologie des philosophes » mérite d'être reconsidérée.

Céline Spector (Université de Bordeaux 3).

54 Sur la «réponse» qu'il convient de donner à Durkheim qui écrit la «politicae scientiae» de Montesquieu, voir B. Karsenti, «Politique de la science sociale. La lecture durkheimienne de Montesquieu », Revue Montesquieu, $\mathrm{n}^{\circ}$ 6, 2002, p. 33-55. 